\title{
Towards a Clinical Efficacy Evaluation System Adapted for Personalized Medicine
}

\author{
Bing Yuan (D) \\ HK Modern Chinese Medicine R\&D \\ Center, Hong Kong
}

\section{Video Abstract}

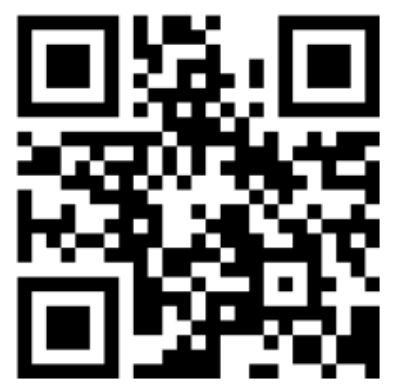

Point your SmartPhone at the code above. If you have a $Q R$ code reader the video abstract will appear. Or use: https://youtu.be/wPcmNzYBI2c
Correspondence: Bing Yuan

HK Modern Chinese Medicine R\&D

Center, Room 2, 7/F Kiu Kin Mansion,

566-568 Nathan Road, Kowloon,

Hong Kong

Email greenisland@vip.I63.com

\begin{abstract}
The rise of precision medicine (PM) has initiated the transition of mainstream medicine from disease-based medicine to personalized medicine, alongside which the US FDA has begun to establish a clinical trial and efficacy evaluation (CTEE) system compatible with personalized medicine based on biological markers. Outside of modern medicine, however, there has always existed a personalized medical system, traditional Chinese medicine (TCM), that is, a personalized medical system built at the organism level with a similar concept and method to today's complexity science. However, under the current CTEE system, TCM has not usually been shown to be effective. The CTEE system of modern medicine has now begun to embrace personalized medicine at the microlevel. Therefore, there is no reason to continue to reject TCM, which is a type of personalized medicine at the organism level. This paper analyzes and compares the commonality and differences between a personalized medical system based on biomarkers established by PM and a personalized medical system based on syndromes in TCM; the results clearly reveal structural relationships between the two medical systems. On this basis, through rigorous logical reasoning, the feasibility of applying the CTEE method which is used in PM to evaluate the efficacy of TCM treatments is demonstrated. The relationship among biomarkers by which PM describes personalized states and modern medical diseases and the relationship among TCM syndromes and diseases are completely consistent. Because of this consistency, the new CTEE system established by the US FDA to promote the development of PM is fully applicable to the clinical trial and efficacy evaluation of TCM treatment methods. Clinical trials and efficacy evaluations based on this system can scientifically prove the effectiveness of TCM, and TCM is expected to be incorporated into the modern medical system based on scientific norms.
\end{abstract}

Keywords: clinical trials, personalized medicine, precision medicine, efficacy evaluation, holistic medicine, basket trial, umbrella trial, Chinese medicine, TCM

The International Classification of Diseases (ICD), an international disease classification system that is currently accepted, is a multiaxis system based on the etiology, location, pathology, and clinical manifestations of disease. For decades, the clinical trial and efficacy evaluation (CTEE) of treatment methods in modern medicine has been primarily based on this disease classification system.

In recent years, natural therapy and traditional medicine have become accepted to a limited extent as supplemental and alternative medicine. Because the concept of evidence-based medicine is widely recognized, reproducible evidence has become a sufficient condition for incorporating medical experience and treatment into modern medicine. In this context, some therapeutic methods and experiences with clinical and practical significance in natural therapy and traditional medicine 
have been accepted and added to the treatment system utilized by modern medicine. Some of the elements that reflect these treatments and experience characteristics have been gradually incorporated into the CTEE system of modern medicine. As a result, the CTEE system based entirely on disease-based medicine has been gradually changing.

However, until the first few years of this century, this change had not jeopardized the core position of the disease classification under CTEE systems. With the rise of PM in recent years, the situation seems to be different. Two initiatives of the FDA have aroused widespread concern in the medical community. First, to accelerate the approval of innovative medicines related to PM, two types of innovative clinical trials have been initiated: Basket Trials and Umbrella Trials. Second, the first anti-cancer therapy that differentiates a tumor not by its source but by abnormal biomarkers was approved in May 2017.

What kind of information underlies these landmark events? Is the CTEE system of today undergoing a quiet but fundamental change? What kind of CTEE system do we need to establish in order to adapt to the transition from disease-based medicine to health-oriented medicine and personalized medicine?

\section{Subtle Changes in the CTEE System Triggered by PM}

The rise of PM started the process of transforming mainstream medicine from disease-based medicine to personalized medicine and will inevitably conflict with the CTEE systems that are based on disease-based medicine. In recent years, to support the development of innovative medicine and related drugs, several initiatives of the US Food and Drug Administration (FDA) have attracted widespread attention in the medical community:

1. Currently, the FDA divides innovative clinical trials into two broad categories. A Basket Trial includes different cancers with the same target genes in one basket to facilitate drug development for tumors of different origins, but with the same target genes. An Umbrella Trial includes a given disease with different target genes (such as lung cancer) under the same umbrella to promote the development of drugs targeting tumors of the same origin but with different target genes. The establishment of these two types of clinical trials means that cancer patients will be able to use effective therapeutic drugs sooner in the future without the long wait time of 7 to 10 years that was common in the past. ${ }^{1}$

2. In May 2017, as a milestone, the FDA approved the first antitumor therapy that differentiates tumors according to biomarkers and not the origin of the tumor. KEYTRUDA (pembrolizumab), which was developed by Merck (MSD), was approved for patients with solid tumors with microsatellite instability-high (MSI-H) or mismatch repair deficiency (dMMR). Previously, the FDA only approved anticancer therapies based on the site-specific origin of a tumor (such as lung cancer or breast cancer).

MSI-H and dMMR are two kinds of common genetic abnormalities. In tumors with these two types of genetic abnormalities, intracellular DNA repair mechanisms are often affected and fail to function properly. Tumors with these abnormalities can appear in multiple sites, such as colorectal, endometrial, and gastrointestinal tracts as well as the breast, prostate, bladder, and thyroid. Therefore, distinguishing these cancers by their genetic variations rather than the site of the disease can result in significantly better treatment outcomes, which has been confirmed during clinical trials. However, if the previous CTEE system were to be applied, the clinical significance of the drug would be difficult to show based on the resultant statistics. $^{2}$

The innovation of the prevailing CTEE system by the FDA is based on following advances in PM:

1. The classification of diseases and their subdivision based solely on tumor origin are usually not sufficient to accurately define the scope of the application of a drug. The introduction of biomarkers such as genes, proteins, and metabolites to describe the individualized characteristics of patients can greatly improve the accuracy of drug selection, which is precisely the reason for the rise of PM.

2. In many cases, individual patient characteristics are not defined by a single biomarker but by a combination of biomarkers.

3. The targets of these biomarkers and their combinations are often associated with more than one specific disease; for example, tumors associated with MSI-H/dMMR may occur in the colorectal, endometrial, and gastrointestinal tracts as well as the breast, prostate, bladder, thyroid and other tissues. 
These studies show that the significance of biomarkers used in PM for labeling targets may not be limited to the fine-grained distinction of diseases in modern medicine, and in many cases, their relationship with diseases is a many-to-many network relationship. In other words, the collection of these targets might eventually form a new body state description system, in parallel with disease classification.

The revision of the CTEE system by the FDA to advance the development of PM has objectively opened a door. With the advancement of PM research and the constant discovery of new targets and target drugs, a description and regulation system of health status that is different from disease classification may occur under the modern medical architecture. This is a huge target and target-drug system based on biomarkers, and it will be an independent system that differs from the disease classification system. As a result, modern medicine will enter an era when either target therapy is at the core, or diseasespecific treatment is combined with target treatment.

\section{Chinese Traditional Medicine: A Personalized Medicine System Missed by Modern Medicine}

In fact, this is not the first time that the CTEE system has encountered such a conflict. Previously, however, the two sides of the conflict were not as fortunate as they are today, and our medicine therefore missed the opportunity to embark on the correct path of development.

In August 2017, an incident that was also related to the US FDA received a great deal of attention in mainland China. The application for Compound Danshen Dripping Pills submitted by the Tasly Corporation of Tianjin, China, received a response from the FDA. The clinical value and statistical significance of the drug were confirmed; however, before the approval of this new drug, a statistically significant six-week-long confirmatory clinical trial is needed. Thus, even after the difficult experience of the FDA application over the past 20 years, marketing approval for Compound Danshen Dripping Pills will still be difficult during the next few years due to the need for a Phase III clinical trial. ${ }^{3}$

The reason that the FDA application for Compound Danshen Dripping Pills is of broad interest in China is that it is the first registered drug application for a Chinese herbal medicine. Due to their remarkable curative effect, Compound Danshen Dripping Pills are a household name in China. Compound Danshen Dripping Pills are one of the rare proprietary Chinese medicines suitable for use in specific diseases in modern medicine. There also exists a classification system for diseases in TCM, but in most cases, the description of the effects of Chinese patent medicines is based on the special syndrome system of TCM.

The relationship between syndromes in TCM and disease is exactly the same as the relationship between targets in PM and disease based on the ICD. Compound Danshen Dripping Pills, which exhibit a high correlation with specific diseases (coronary heart disease), have experienced a difficult course towards approval for nearly 20 years and have not yet been registered as a drug. For other Chinese patent medicines that are less relevant to specific diseases, it is more difficult to show their clinical significance under the CTEE system based on disease-based medicine. In TCM, there are many Chinese patent medicines that have undergone numerous human trials in China over thousands of years and have shown good results in many diseases for which modern medicine is ineffective. Since these Chinese patent medicines cannot yet pass the CTEE system based on disease-based medicine, none of them have been utilized in mainstream medicine.

In 1956, during an outbreak of Japanese encephalitis in mainland China, the well-known doctor of TCM Mr. Pu Fuzhou treated 167 patients with Japanese encephalitis, none of whom died. However, this significant clinical outcome cannot be recognized as the result of medical research, even in mainland China. The reason is simple: 167 patients were administered 98 prescriptions, and each prescription was given to an average of fewer than two people. Thus, the treated patients belong to individual cases that show no statistical significance. ${ }^{4}$

Today, the same problem is faced in the medical response to the flu. In TCM, the various flu types, including SARS, avian flu, and influenza A, are generally divided into different types of syndromes, such as windcold, wind-heat, heat transformed from wind-cold, and wet entrained wind-heat, according to the clinical manifestations of the patients at onset, rather than the viruses involved. However, personalized treatments based on syndrome differences often yield good results. The correspondence between the syndrome and treatment regimen is seen everywhere in books on TCM. Here, we see that the relationship between TCM syndromes and various flu strains is similar to the relationship between biomarker abnormalities, such as MSI-H and dMMR, and multiple 
tumors of different sources. When considering diseases caused by nonmicrobial pathogens, such as cardiovascular and cerebrovascular diseases, metabolic syndromes, and tumors, the treatment system of TCM based on syndrome differentiation represents a complete response system, from diagnosis to treatment.

As mentioned above, without FDA reform, KEYTRUDA is unlikely to be approved for marketing based on the original CTEE system of disease-based medicine. This is fortunate for KEYTRUDA because people have now realized the limitations of disease-based medicine and have begun to explore new personalized medical systems. Similarly, if we apply similar principles to the CTEE of Chinese herbal medicines for the treatment of influenza, we can also obtain scientific proof of the effectiveness of these herbal formulas.

The world has always viewed TCM with skepticism. Modern medicine has developed advanced detection instruments and therapeutic equipment as well as advanced pharmaceutical technologies, including chemical synthesis and plant extraction methods. However, only a few diseases can truly be treated with drugs at present, and there are always side effects that cannot be avoided. In the case of most diseases for which modern medicine is not effective, using TCM methods can often yield good treatment results. As modern medicine begins to reform the CTEE system based on the problems faced by PM, might we realize that due to the limits of the development level of modern science and medicine and associated prejudices, mainstream medicine has been still rejecting a personalized medical system that is much more effective in coping with the complexity of life than modern medicine including future precision medicine (PM)?

\section{PM and TCM: Two Personalized Medicine Systems at Different Levels}

PM has started the long journey towards building a personalized medical system. However, after thousands of years of countless human trials, our Chinese ancestors left the modern world with a fairly complete personalized medical system. The study of PM is currently limited to biomarkers based on genetic variation, and the short-term goal is focused on cancer. With the advancement of PM, research on biomarkers has gradually expanded to proteins and metabolites and to even more extensive fields. Considering the ICD system, which includes more than
29,000 diseases, completion of the exploration of tumors is only the first step on this long road. One day, even if we have fully grasped these 29,000 diseases, we still have to face the integration of treatments when multiple diseases occur at the same time. In this context, can these precise drugs that have been developed for a single disease and have different side effects be applied simultaneously in one patient?

In Europe and North America, many elderly patients currently take up to 20-30 kinds of drugs every day. However, this does not improve their health status and instead causes many drug-induced diseases. As a result, the United States has introduced a policy that limits the number of drugs to be taken simultaneously to nine.

The emergence of PM is the inevitable result of the gradual deepening of modern medical research along the order from organ, tissue, cell to molecular. Molecules are the smallest components with characteristics of life. The establishment and development of molecular biology indicated that modern scientific research on life had extended to the last layer. Further research based on the concept of analytical science is, on the one hand, horizontal expansion at the molecular and cellular levels; on the other hand, integration from the molecular and cellular level to the organismal level. Current research in PM is the horizontal extension of modern medical research at the molecular level. At the same time, biologists and medical scientists have realized that life cannot simply be attributed to the interaction between molecules that make up life. To understand the holistic characteristics of life and to effectively control the whole status of an organism, biology and medicine will have to go toward integration. In recent years, the rise of systems biology and the increased voice of medical integration have reflected this general trend in the development of life sciences and medicine.

However, can biology and medicine achieve holistic integration based on microscopic analysis? As the applied fields of the natural sciences, biology and medicine have been driven by advances in natural science methods and techniques for the last several hundred years. Today, along with the order from molecules to atoms and elementary particles, the revealing of natural laws through science has deepened to a more microscopic level. On the other hand, with the evolution of scientific ideas and methods, people no longer attribute the problems of complex systems, such as the universe, the earth, the ecology, the human body, and the human brain, to the problems of simple systems. Instead, science has begun to face complexity directly and 
to approach research according to the original face of complex systems.

The rise of complexity science has triggered a fundamental change in scientific concepts and ways of thinking. The most important concept introduced by complexity science is "emergence". Due to the existence of the "emergence" phenomenon of complex systems, researchers cannot deduce the macrostructure or nature of the system from its microlevel structure and behavioral rules. The method of summing up the knowledge of the parts is essentially inappropriate to describe holistic emergence because emergence no longer exists when the whole is broken down into parts. ${ }^{5}$ With the characteristics and laws of the "emergence" of complex systems were revealed, scientists realized that the whole can lead to the microlevel through analysis, but integration based on the results of analysis is difficult to achieve. In other words, it is impossible for modern medicine to achieve integration on the basis of analysis through the "analysisreconstruction" method and to ultimately grasp the human body as a whole.

Systems biology, which was established based on the early "analysis-reconstruction" concept of systems science in the 1970s-1980s, has now spanned more than 30 years in the integration studies of organisms. However, what it has brought to people is not the wonderful prospect of integration, but the helplessness of scientists facing the complexity of life: the complexity of biological systems far exceeds the imagination of people, and current science is not suitable for studying the entire biological system. These studies can only start from "small systems" that have a certain function and are relatively independent. ${ }^{6}$ The adaptability of organisms makes systems biologists more confused: repeatability is the basic principle of natural science research; however, due to the adaptability of organisms, when scientists repeatedly test certain phenomena and processes that occur in living organisms, reproducibility of the test results in the strict sense often becomes a "luxury" requirement. Adaptability is a natural feature of living organisms and an objective existence that science cannot change. Therefore, the problem can only be attributed to the way in which we study it. That is, the research methods currently used in systems biology are fundamentally unsuitable for studying life with complex adaptability.

Complexity science is formed by the convergence of some transverse disciplines, such as systems science, cybernetics, information theory, dissipative structure theory, and catastrophe theory. Complexity research has passed its early stages. With the discovery of "emergence" phenomena and laws, and the "analysis-reconstruction" method has repeatedly hit the wall in the practice for the integration of complex systems, scientists have gradually realized that since complex systems cannot be understood and captured through analysis-based reconstruction, they can only be taken as a whole and their functional model can only be established by investigating their input and output. With such a model, people can intuitively understand the internal laws of complex systems and achieve their overall regulation.

Today, when we view TCM with thousands of years of history from the commanding heights of complexity science, we find that the approach by which the theoretical models and state description systems of TCM were established is strikingly consistent with the holistic approach to complexity science. The establishment of personalized state description, TCM is based on the holistic level, while PM begins at the molecular level. Modern medicine is aware of the limitations of disease-based medicine and is beginning to establish a personalized state description, which undoubtedly represents a great advancement in medicine. However, the establishment of state description at the microlevel will certainly lead to problems that need to be integrated into the whole but are difficult to integrate. TCM directly establishes state descriptions from the holistic level, so there is no integration from the microlevel to the whole, and the integration that it faces is further upward integration based on the elements of the holistic level.

To describe a variety of personalization statuses, TCM has introduced only approximately 100 state variables (known as syndromes in TCM). However, recent genetic studies have shown that the number of human genes (including coding and noncoding genes) has reached approximately $40,000,{ }^{7}$ and the number of genetic mutations is much larger than this. With the progress of omics research on proteins, metabolites, etc., the discovery of abnormal biomarkers will reach a larger scale. The number of state variables in PM state description system based on biomarkers will obviously far exceed the scale of TCM. The integration of medicine includes integration from the microlevel of PM to the whole, and integration based on elements at the whole level of TCM involves the integration of complex diseases with multiple TCM syndromes.

In recent decades, studies using modern analytical methods on TCM syndromes have shown that the 
appearance of a syndrome in TCM is often accompanied by multiple abnormal biomarkers at the microscopic level and that effective treatment of these syndromes is also often accompanied by the recovery of these abnormal biomarkers. In turn, focusing on the microscopic level, one cannot expect to achieve the macroscopic effect of returning abnormal syndromes to normal states by accurately treating multiple abnormal biomarkers corresponding to the syndrome. ${ }^{8}$ The reasons are as follows: 1 ) it is difficult to find all the microscopic biomarkers associated with the syndrome; 2) it is difficult to find accurate drugs that have an adjustment effect on these biomarker abnormalities; 3) these precision drugs not only act on target biomarkers but may also produce other effects or side effects in the human body. The combined effects of a variety of such "precise" drugs on the human body are unlikely to match our expectations.

Based on the state description of the holistic level established by TCM, the direct controlled point of drug research is the syndrome at the holistic level. Thus, treatment of disease through TCM does not involve integration from the microscopic to the holistic level. Since the total number of syndromes in TCM is only approximately 100 , when multiple syndromes are diagnosed at the same time, it is usually necessary to analyze the importance and harm of each syndrome related to human functions and diseases to determine their prioritization for treatment. Therefore, the number of syndromes that a treatment plan needs to cope with does not exceed 10 in most cases, and such complexity is much lower than that of integration from the microlevel to the whole.

When we clarify the structural relationships among PM, systems biology, and TCM under the life sciences framework as well as the hierarchical relationship between the personalized medical system established based on PM and the TCM syndrome system, along with their practical significance in medical integration, it is not difficult to understand the profound methodology underlying the surprising clinical efficacy of TCM.

Today, the reform of the CTEE system by the FDA opens the door for PM to establish a personalized state description from a microscopic level and confirms the profound scientificity and advanced nature of TCM's personalized state description established at the holistic level from a certain perspective. The question is whether this door can be opened further; that is, whether the evaluation of the new CTEE system for personalized medical methods can be extended from the micro level to the holistic level in order to evaluate drugs based on the TCM syndrome system. With this opportunity, modern medicine will simultaneously begin the historical process from analysis to integration while moving towards personalized medicine.

\section{Problems Faced by PM in Establishing a Personalized Medical System}

As the introduction of biomarkers expands to a wider range of fields, involving proteins, metabolites, etc., and clinically significant biomarkers and targeted drugs are continuously discovered, the following questions will arise in the process of establishing personalized state description and regulation systems in PM:

1. Many targets are determined not by a single biomarker, but by a set of biomarkers. If targeted drugs are used to distinguish the targets, it may sometimes be found that more than one or a group of biomarkers can be treated with the same target drug. For example, KEYTRUDA can be used for two types of abnormal biomarkers, MSI-H and dMMR. That is, biomarkers corresponding to the same target drug or therapy will also need to be brought together in a collection, and it is therefore necessary to introduce a concept similar to the syndrome in TCM. Since the target system may form an independent system juxtaposed with the disease classification system, its appearance will also be accompanied by a series of problems, such as how the system builds its architecture, how these target collections are named, and what kinds of structural relationships exist among these targets and target sets.

2 . With the accumulation of clinically significant biomarkers, some of the biomarkers (or combinations thereof) will be found have similar diagnostic meanings; expressed in the mathematical language, this means that they are linearly related. Therefore, PM must also organize the biomarker system in a structured way and optimize it by removing redundancy, so that it can be gradually transformed into a "simplest applicable" system composed of biomarkers that are relatively easy to detect and exhibit high diagnostic accuracy.

3. In general, more than one drug can be screened for one target, and the effect of one drug is not limited to only one target. After the target system reaches 
a certain scale and develops a certain structure and we find that a group of (or one) biomarker abnormalities can be treated with a previously discovered targeted drug, we must first distinguish whether the group of (or this one) biomarkers should be included in the biomarker collection corresponding to the target drug or a new therapeutic effect of the target drug has been found. This means that the classification of targets may involve different schemes and layouts, and the boundaries between targets may be blurred, similar to the syndrome system of TCM.

The above problems can be dazzling at first glance, but this is a necessary process for any complex real system establishing state description. The multiplication of factor numbers and complexity caused by the downward shift in the level of research focus makes this process in the human body more complex and requires more workload. Establishing the state description is the first problem that must be solved by natural science to study an objective object. This process can usually be attributed to the introduction of a group of state variables that have a certain degree of completeness and independence, and through statistical analysis based on investigation of the inputs and outputs of the system to establish the relationship among these state variables.

If the state variables introduced by PM to establish the state description correspond to the target point, the target point to which we refer here may be a single biomarker, a combination of multiple biomarkers, or a set containing multiple biomarker combinations. Completeness here refers to the ability to fully describe all possible pathological states of the human body. Because PM research involves expansion in the horizontal direction and integration from the bottom up, it is impossible to obtain a toplevel design based on completeness. Instead, in the course of this research, with the introduction of new biomarkers for new for new pathological conditions, the completeness of the coverage of the target system will naturally be improved over time.

Independence is a concept that is contrary to relevance. The greater the correlation between variables, the smaller the independence of the variables. A full correlation means that two variables are equivalent and that either of them can be completely expressed by the other. In fact, it is very difficult to find a set of completely independent state variables to describe the human body as a whole organism. In reality, scientists always try to find some state variables with less relevance and gradually replace the more relevant variables as research progresses. Description of the system based on a set of state variables with a higher degree of correlation will inevitably lead to duplication or redundancy, increase the complexity of the structure, and reduce the accuracy of the state description.

In the personalized state description system established by PM, target points as state variables are the core of the system, which are equivalent to the syndromes of TCM. There are intricate multiple associations among the targets and associated biomarkers it contains as well as their combinations. Of course, in some special cases, the target may consist of a combination of biomarkers or a single biomarker. In the state description system, the independence of the target is critical. Therefore, in the research process, relevant analyses should be conducted in a timely manner whenever new biomarkers (or biomarker combinations) are introduced in order to remove redundancy and optimize the system. As a result, the personalized state description and regulation system constructed under PM cannot simply consist of accumulation of the increasing number of biomarkers and their target drugs; instead, it should be a structured and applicable system that better satisfies completeness and minimizes the scale as much as possible.

Complex targets are usually defined by multiple abnormal biomarkers (or combinations thereof). Diagnosing such targets requires not only qualitative rules but also quantitative data. Therefore, based on statistical analysis, the degree of association between abnormal biomarkers (or their combinations) and the target can be used as a quantitative basis for judging whether or not the target is established, and the correlation between the biomarkers corresponding to the same target is the basis for classifying the biomarkers. If the correlation between two biomarkers is very high (ie, they exhibit little independence), it is reasonable to remove a biomarker with a lower degree of relevance to the target or to select only one of the diagnostic values in order to avoid double counting. Therefore, the biomarker collection corresponding to the target can be classified, structured and optimized so that the target diagnosis is more simplified and accurate from both qualitative and quantitative perspectives.

The study of PM is currently in the early stages of accumulating targets and targeted drugs. With the rapid growth of the number of targets and target drugs, dealing with the structure and relationships among them will become an increasingly prominent problem. To capture 
the correlations among very large numbers of biomarkers, big data analysis and machine learning technology must be used to comprehensively collect patient health information, such as gene, protein, and metabolite data, and conduct large-scale statistical analysis. The "All of Us Research Program" of American PM has begun work based on this research approach. With the development of PM research, new challenges for leaders in PM including the FDA and the NIH will be that according to the new problems, constantly improve the rules of the CTEE system, optimize the target system, and promote the establishment of a structured and standardized target system.

\section{Problems Faced in Establishing a CTEE System Suitable for TCM}

Different from PM, for which the personalized state description system was established through the horizontal expansion of targets (state variables), TCM's state description system was established from the top down. The "visceral manifestations" theory of TCM is essentially a functional model of the physiological and pathological activities of TCM. At the core of this theory are organs called Zang-organs or Fu-organs, both of which have certain physiological functions. Various organs and all parts of the human body are connected as a whole through a network referred to as meridians. The Qi, blood and fluid that flow in the meridians play a role in supplying energy and nutrition to maintain the functional activities of various parts of the body. The functional states of these viscera and basic life matter are expressed by the introduction of certain state variables, and these state variables together form the state space of the human body. The abnormal state of each state variable corresponds to a syndrome of TCM and is expressed by a corresponding set of symptoms and signs. Obviously, since the state variables are introduced based on the human body model established from the overall level, the TCM state description system is characterized by a top-down, top-level design. The comprehensive coverage of various functions and physiological activities in the human body model ensures the completeness of the state variable system.

Today, based on such a state description system (ie, the syndrome differentiation and treatment system of TCM), a doctor of TCM can always find out where the patient's abnormal state and provide a personalized treatment plan in the vast majority of cases, regardless of whether that patient has been given a modern medical diagnosis of a specific disease. Thus, the completeness of the TCM state variable system is also demonstrated from a practical perspective. Of course, there are also cases in which patients are diagnosed with certain diseases based on modern medicine but a doctor of TCM cannot make diagnosis because it has not yet manifested in symptoms and signs. This is precisely the case in which TCM needs to improve its state description system further through the introduction of new testing indicators.

At present, approximately 100 syndromes are used in the state description of TCM, which is quite an insignificant quantity. However, even if no more than 5 syndromes appear at a time, the number of combinations of states that can be described by this system can reach 10 billion, and the number of combinations of states that appear in no more than 10 syndromes at a time is a more unimaginable astronomical. This shows that the TCM syndrome differentiation and treatment system is actually a personalized state description and regulation system on a scale beyond the imagination, but it is much simpler than the personalization state description system of PM.

Due to the limitations of scientific concepts and the development level of science and technology, TCM has been excluded from mainstream medicine, and no rigorous and scientific CTEE system has been formed to promote its development. Today, the reform of the CTEE system triggered by $\mathrm{PM}$ has removed the conceptual barriers to the acceptance of TCM by mainstream medicine. Therefore, it presents realistic possibilities for establishing a set of CTEE rules suitable for TCM therapies.

The Basket Trial and Umbrella Trial designations developed for PM are completely suitable for CTEE of the TCM syndrome differentiation and treatment system. A Basket Trial can put patients who have the same syndrome but are diagnosed with different diseases into the same "basket" to promote the development of drugs and formulations for treating the syndrome. An Umbrella Trial groups various types of syndromes (including combinations of syndromes) of a disease under the same "umbrella" to systematically study different treatments for the same disease based on different syndrome types. Thus, the identification of the type of syndrome associated with a disease is no longer based entirely on experience but may also involve rigorous statistical analysis.

The "basket test" and the "umbrella test" greatly expand the scope of a clinical trial from one disease to multiple diseases or from one disease type to multiple disease types. It is obviously not sufficient to allocate 
a sample size originally required for a clinical trial to multiple diseases associated with a syndrome, or to multiple syndrome types associated with a disease. Accordingly, it is necessary to lengthen the trial period and expand the sample size, so that the sample size required for statistical analysis can be obtained for each disease associated with a syndrome and each syndrome associated with a disease.

The CTEE system of PM requires the establishment of a classification system that reflects the structural relationships of the target. Similarly, the CTEE system of TCM needs to establish a structured syndrome classification system that conforms to scientific norms. The state description of TCM is performed in a top-down manner, with a top-level design. Structured is the scientific and standardized reconstruction and organization of the toplevel design. In TCM, there are four systems of syndrome differentiation and treatment. In addition to the abovementioned syndrome system based on the theory of "visceral manifestation", there are three other syndrome-based systems for treating epidemic and infectious diseases such as wind-cold, warm-heat and damp-heat disease, ie, Six Meridians syndromes, Wei-qi-ying-xue syndromes and Sanjiao syndromes, similar to the system of relativity at the astronomical level and the system of quantum mechanics at the microlevel in theoretical physics. The structuralization of the syndrome differentiation and treatment system first requires the integration of the four systems into one and builds a unified system conforming to strict rules of logic. As a top-level design, the new system should be able to cover all the syndromes of the original four systems and ensure the relative independence of each syndrome and rigorous logical structure among the syndromes.

The syndromes of TCM are defined by symptoms and signs. Of course, to improve the level of objectivity of syndrome diagnosis, some test indicators can be introduced on the basis of statistical correlation, which include biomarkers showing a good correlation with TCM syndromes. In the collection of symptoms, signs, and indicators that are diagnostically significant for syndromes, the association of symptoms, signs, and test indicators with syndromes does not show a one-to-one correspondence in most cases. Therefore, regarding the standardization of syndrome diagnosis, there must be a problem of quantifying the diagnostic significance of different symptoms, signs, and detection indicators based on correlation. Achieving an accurate identification of syndromes requires not only qualitative rules but also quantitative calculations. This problem also arises when a combination of multiple biomarkers is required to determine a target of PM.

Whether a syndrome of TCM or target of PM is considered, an accurate diagnosis obviously not only needs to clarify the qualitative conditions for the establishment of the diagnosis but also needs to determine the quantitative data expressing the degree of correlation. The acquisition and continuous optimization of these precise data are based on the statistical analysis of large sample cases. Today, the continuous advancement of computer data analysis and artificial intelligence machine learning technology provides a broader imaginary space for the integration of clinical trials and clinical application processes as well as the establishment of new therapeutic evaluation mechanisms. At the same time, it also poses new challenges for the rules of the CTEE system and the policy of supervision.

\section{Concluding Remarks}

The life sciences of the 21 st century are moving towards integration and personalization. TCM is precisely the medical system that embodies overall integration and personalization and has been verified by clinical practice over thousands of years. In order to adapt to the trend of personalized medicine, modern medicine has made fundamental reforms to the CTEE system that had been implemented for a long time. The milestone significance of this reform lies in the fact that some targeted drugs developed by PM, which did not have clinical effect based on the previous CTEE system, now have a significant effectiveness in accordance with the new CTEE system. However, the greatest significance of the new efficacy evaluation system not yet known by its implementers is that it can also be used for the evaluation of the efficacy of TCM. Under this evaluation system, the extensive effectiveness of TCM will be scientifically proven.

The development of PM will undoubtedly bring huge advances in disease detection, diagnosis and treatment. However, it still cannot escape the limitations of reductionist methods, and therefore is unable to achieve the overall integration required by medicine. The introduction of this new CTEE model into TCM means that in the near future, TCM will be incorporated into the modern medical system in a scientific manner. As a result, mainstream medicine will not only shift from disease medicine to personalized medicine, but also enter the era of holistic medicine characterized by integration. 


\section{Abbreviations}

PM, precision medicine; CTEE, clinical trial and efficacy evaluation; TCM, traditional Chinese medicine; ICD, International Statistical Classification of Diseases.

\section{Data Sharing Statement}

Not applicable.

\section{Ethics Approval and Consent to Participate}

Not applicable.

\section{Consent for Publication}

Not applicable.

\section{Acknowledgment}

Thanks to the American Journal Experts for retouching this article.

\section{Authors' Information}

The author graduated from the Beijing University of Chinese Medicine in 1983 and subsequently studied undergraduate courses in computer software design. He has background knowledge and research experience in modern medicine, traditional Chinese medicine, philosophy of science, complexity science, artificial intelligence, etc. and has more than 40 years of clinical experience in integrating Chinese and Western medicine. The author has long been engaged in the research of history and methodology of Eastern and Western medicine. In recent years, he has been paying attention to the research progress in the frontiers of life sciences such as precision medicine, systems biology, integrated medicine and constitution medicine. He is dedicated to exploring trends and methodologies in modern medicine and biology and has published a series of influential academic papers and treatises. His recent representative work "Towards Holistic Medicine" will be published by the CRC Press soon.

\section{Funding}

There is no funding to report.

\section{Disclosure}

The author reported no conflicts of interest for this work.

\section{References}

1. West HJ. Novel precision medicine trial designs: umbrellas and baskets. JAMA Oncol. 2017;3(3):423. doi:10.1001/jamaoncol.2016. 5299

2. FDA News Release. FDA approves first cancer treatment for any solid tumor with a specific genetic feature. 2017. Available from: https:// www.fda.gov/newsevents/newsroom/pressannouncements/ ucm560167.htm. Accessed September 29, 2018.

3. China Fund News. Tasly's "compound danshen dripping pill" applied for 20 years into the US to enter the sprint phase. 2017. Available from: http://finance.ifeng.com/a/20170831/15632737_0.shtml. Accessed April 13, 2021.

4. Tang R.. Chinese medicine really cured Japanese encephalitis in 1954 and 1956? Available from: https://richardfx.blogspot.com/2010/11/ 19541956.html. Accessed November 7, 2010.

5. Xinrong H. Complexity Science and Philosophy. Beijing: Central Compilation Press; 2007:150-175.

6. Taijiao J, Yanhong X, Tao X. Systems biology: a new field of biological science. Prog Biochem Biophysics. 2004;31(11):957-964.

7. Willyard C. New human gene tally reignites debate. 2018. Available from: https://www.nature.com/articles/d41586-018-05462-w. Accessed April 13, 2021.

8. Yuan B. On the way to status medicine: a medical revolution triggered by precision medicine. China J TCM Pharm. 2017;32(4):1434-1448.

\section{Publish your work in this journal}

Pharmacogenomics and Personalized Medicine is an international, peer-reviewed, open access journal characterizing the influence of genotype on pharmacology leading to the development of personalized treatment programs and individualized drug selection for improved safety, efficacy and sustainability. This journal is indexed on the American Chemical Society's Chemical Abstracts Service (CAS). The manuscript management system is completely online and includes a very quick and fair peer-review system, which is all easy to use. Visit http://www.dovepress.com/testimonials.php to read real quotes from published authors. 